\title{
Aqueous Two-Phase System Combined with Ultrasound for the Extraction of Polysaccharide from Hawthorn (Crataeguspinnatifida) Leaves
}

\author{
Ri-li HAO, Juan-juan MA, Kun LI, Chuan-he ZHU ${ }^{a,{ }^{*}}$ and Xiao-cun \\ ZHANG ${ }^{\mathrm{b}, *}$ \\ College of Food Science and Engineering, Shandong Agricultural University, Tai'an, \\ 271018, PR China \\ achhzhu@sdau.edu.cn, bxczhang@sdau.edu.cn
}

Keywords: Hawthorn Leaves, Polysaccharide, Aqueous Two-Phase System, Ultrasound, Response Surfacemethodology.

\begin{abstract}
Aqueous two-phase system (ATPS) combined with ultrasonic technology was employed to extract polysaccharide from hawthorn leaves in this study. The partition behaviors in different ATPS were investigated first, polyethylene glycol (PEG) $11.5 \%$ and (NH4)2SO4 14\% were selected as the optimum solvent for extracting polysaccharide. Using the optimum ATPS solvent, the ratio of material to solvent, The time, temperature and power of the ultrasonic treatment were investigated using a single factor experiment and Box-Behnken Design (BBD) of response surface methodology (RSM) to obtain the optimum conditions for extracting polysaccharide. The optimum conditions were the ratio of material to solvent 1:34, ultrasonic time (UT) $35 \mathrm{~min}$, ultrasonic power (UP) $300 \mathrm{w}$ and ultrasonic temperature (UTE) $65^{\circ} \mathrm{C}$. With the optimal conditions, the maximum yield of polysaccharide was $3.05 \%$. The present study provided a foundation for further purification of polysaccharide and offered a valuable alternative for extracting polysaccharide from plants.
\end{abstract}

\section{Introduction}

Hawthorn (Crataeguspinnatifida.) belongs to Rosaceaeplants and is cultivated in many areas of China [1]. According to literature, hawthorn leaves and fruits are widely used in China for their pharmaceutical and nutritive value [2]. The pharmacological properties of hawthorn leaves are proven to be dependent on the polysaccharides. Some researchers have recently been attracted to the hawthorn leaves because of their various biological activities [3]. The current studies have shown that polysaccharides have bioactive properties including antitumor, antioxidant, anti-diabetic, immunomodulatory, hepato-protective, anti-hypertensive and hypocholesterolemic activities, which are potentially important for human health [4-5]. Therefore, the polysaccharides of extracts have been considered as additives in food and pharmaceutical applications for their unique bioactive properties and chemical structures [4]. In addition, most of polysaccharidesare high-molecular weight macromolecules that can be easily dissolved and/or dispersed in water.

Recently, some studies have been done on extraction methods of polysaccharide from plants. The conventional extraction methods are related to heating or boiling such as hot water extraction and reflux extraction [6]. Although the conventional methods are convenient and safe, they have disadvantages such as a long extraction time at high temperature, leading to a degradation of polysaccharide molecules and a decrease in their pharmacological activity, and a low product yield [7]. Therefore, 
some new techniques for the extraction of polysaccharide are tested, such as ultrasonic-assisted extraction (UAE), microwave-assisted extraction (MAE), supercritical fluid extraction (SFE), enzyme-assisted extraction (EAE) and aqueous two-phase system extraction (ATPSE). Firstly, UAE and MAE have the advantages of accelerating the extraction process, reducing solvent consumption and high yield of polysaccharide [7], but MAE has the disadvantage of inhomogeneous heating [5]. SFE is a new method of extracting compounds from plants which is costly and requires complicated equipment operation [8]. EAE can improve the purity of polysaccharide under certain conditions, but it requires long time and high temperature. According to the physicochemical properties of polysaccharide such as hydrophili city, the methods of ATPSE have been noticed by researchers. ATPS consists of short chain alcohols/hydrophilic solvents and inorganic salts. It allows to extract compounds from plants with the advantages including lower costs, higher extraction yields, shorter extraction time and higher purity for target compounds in one step [9]. In this study, a combination of ultrasound treatment and ATPS was used to extract polysaccharides from hawthorn leaves.

\section{Materials and Methods}

\section{Plant Materials}

Hawthorn leaves of April and May were collected from Laiwu, Shandong province.

Determination of polysaccharide

Polysaccharide was determined by the phenol/sulfuric acid method using glucose as a standard with ultraviolet spectrophotometer (754N, APL, Shanghai). The yield of polysaccharide was calculated using the following equation [10]:

Yield of polysaccharide $=\mathrm{CV} / 1000 \mathrm{~m} \times 100 \%$.

Where $\mathrm{C}$ is the concentration of polysaccharide $(\mathrm{mg} / \mathrm{ml}) ; \mathrm{V}$ is the total volume of the extraction solution $(\mathrm{ml})$; and $\mathrm{m}$ is the weight of the hawthorn leaves $(\mathrm{g})$.

\section{Partition Behaviors of Polysaccharide in ATPS}

The partition coefficient $(\mathrm{K})$ was calculated by the equation as follows:

$$
\mathrm{K}=\frac{C_{t}}{C_{b}}
$$

where $C_{t}$ and $C_{b}$ were respectively equilibrium concentrations of polysaccharide in the top phase and bottom phase.

\section{Selection of ATPS}

\section{UAATPS Extraction from Hawthorn Leaves}

A sample of $1.000 \mathrm{~g}$ of hawthorn leave powder was macerated in a glass flask with a certain amount of an optimal ATPS. Ultrasonic-assisted extraction was conducted. After the extraction finished, the extract was centrifuged (TD25-WSlow speed centrifuge, Shanghai), and then the filtrate was held for $1 \mathrm{~h}$. The absorbance of bottom phase was determined by phenol/sulfuric acid method. The yield of polysaccharide was calculated by the above equation.

Optimization of UA-ATPS extraction

According to the result of the single-factor experiment, Box-Behnken Design (BBD) with four factorial points, three levels and three central points leading 27 sets of experiments was implemented. The ratio of material to solvent ( X1: 1:30, 1:35, and 
1:40); UT (X2: 30, 35, and 40min); UP (X3:300, 360, and 420w); UTE (X4: 50, 60, and $\left.70^{\circ} \mathrm{C}\right)$.

\section{Statistical}

All experiments were run in triplicates; results were shown as mean values $\pm \mathrm{SD}$. Statistical analysis was conducted with a statistical analysis system (SAS 8.0 software).

\section{Results and Discussion}

\section{Effects of ATPS on the Yield of Polysaccharide and K}

Various ATPS, including ethanol/ $\left(\mathrm{NH}_{4}\right)_{2} \mathrm{SO}_{4}, \mathrm{PEG} /\left(\mathrm{NH}_{4}\right)_{2} \mathrm{SO}_{4}$, propyl alcohol/ $\left(\mathrm{NH}_{4}\right)_{2} \mathrm{SO}_{4}$ and ethanol/ $\mathrm{K}_{2} \mathrm{HPO}_{4}$ were studied for their effects on the yield of polysaccharide and $\mathrm{K}$. The results are shown in Fig. 1. Among these ATPS, an ATPS based $\mathrm{PEG} /\left(\mathrm{NH}_{4}\right)_{2} \mathrm{SO}_{4}$ showed the highest yield and minimum $\mathrm{K}$ of polysaccharide in hawthorn leaves. Based on Fig.1, polysaccharide was easily dissolved in bottom phase and the effect of separation was better if $\mathrm{K}$ was smaller. Then the effect of PEG and $\left(\mathrm{NH}_{4}\right)_{2} \mathrm{SO}_{4}$ concentration on yield of polysaccharide and $\mathrm{K}$ were studied. According to Fig. 2-a, the data indicated that the yield of polysaccharide was increased and $\mathrm{K}$ was decreased with the increase in the concentration of $\left(\mathrm{NH}_{4}\right)_{2} \mathrm{SO}_{4}$ from $12 \%$ to $14 \%$, but the yield was decreased when the concentration exceed $14 \%$. This was most probably caused by the fact that an increase in the concentration of $\left(\mathrm{NH}_{4}\right)_{2} \mathrm{SO}_{4}$ may lead to the volume increase of the bottom phase, and polysaccharide dissolved in that phase. When the concentration of $\left(\mathrm{NH}_{4}\right)_{2} \mathrm{SO}_{4}$ exceed the limits of $14 \%,(\mathrm{NH} 4)_{2} \mathrm{SO}_{4}$ separated out and less free water was available to dissolve polysaccharide. As shown in Fig 2-b, the yield of polysaccharide and $K$ were increased with the increase in PEG concentration from $7 \%$ to $11.5 \%$. When the concentration of PEG exceeded $11.5 \%$, the yield of polysaccharide was decreased and $\mathrm{K}$ was increased respectively. However, excessive $\left(\mathrm{NH}_{4}\right)_{2} \mathrm{SO}_{4}$ was separated as solid when the PEG concentration was increased. According to the yield of polysaccharide and $\mathrm{K}$, the concentration of PEG was selected at $11.5 \%$. Therefore, based on the data from this experiment, PEG (6000) $11.5 \%$ and $\left(\mathrm{NH}_{4}\right)_{2} \mathrm{SO}_{4} 14 \%$ were selected as the optimum ATPS for further study.

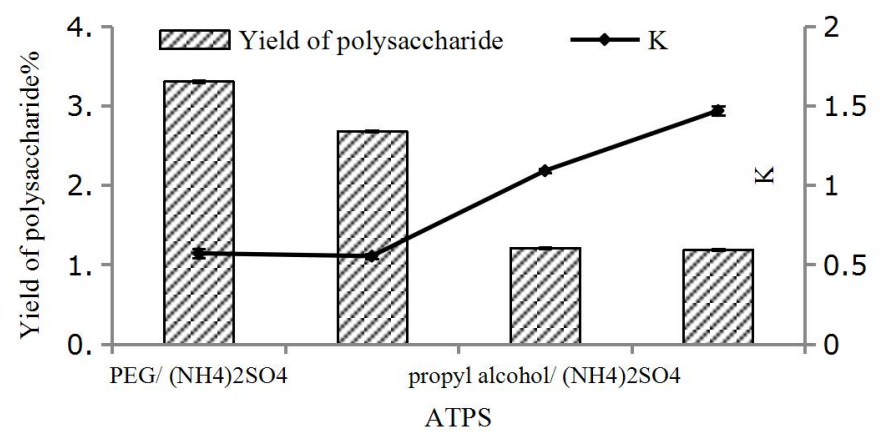

Fig.1. Effect of different ATPS on yield of polysaccharide and $\mathrm{K}$ 


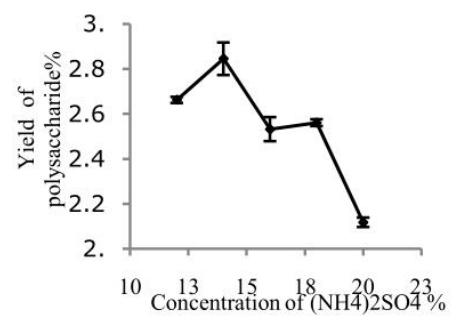

Fig.2-a.Effect of concentration of $\left(\mathrm{NH}_{4}\right)_{2} \mathrm{SO}_{4}$

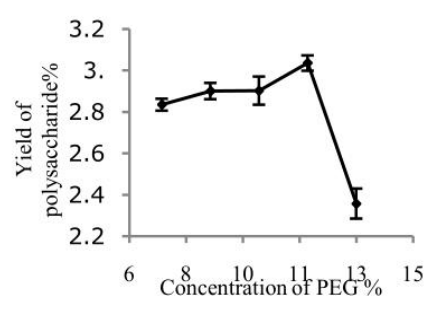

Fig.2-b.Effect of concentration of PEG on yield of polysaccharide yield of polysaccharide

\section{Effect of UA-ATPS Process Variables on the Yield of Polysaccharide}

\section{Effect of the Ratioofmaterial to Solvent}

As shown in Fig 3, the yield of polysaccharide increased gradually with increase in ratioofmaterial to solvent from 1:15 to $1: 35$, but there was no significant change in the yield of polysaccharides as the ratio continued to increase. If the extraction was performed under the high ratio of material to solvent, it required too much amount of heat dissipate in the ATPS and little heat was absorbed by the material. However, the solvent wouldnot be enough for polysaccharide if the ratio was too low. Therefore, the optimum ratio was selected as 1:35.

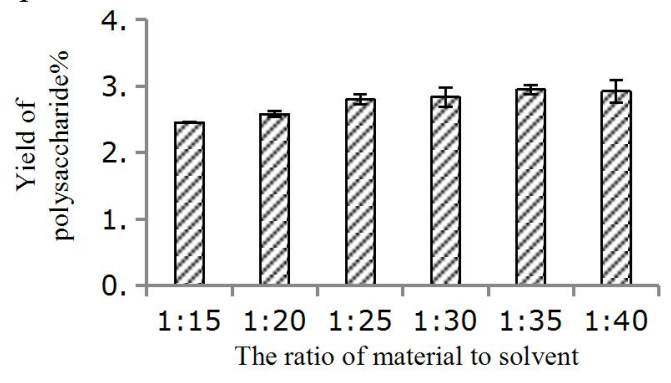

Fig.3. Effect of the ratio of material to solvent

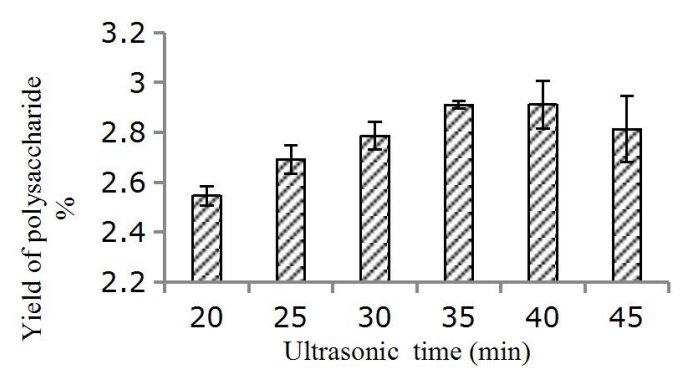

Fig.4. Effect of ultrasonic time on yield of polysaccharide on yield of polysaccharide

\section{Effect of UT}

The result has been shown in Fig 4, and it could be found that the yield of polysaccharide was increased with the increase in time from 20 to $35 \mathrm{~min}$, and then was decreased gradually when the time exceed $35 \mathrm{~min}$. Ultrasound can facilitate the release of polysaccharides from plant cells to the liquid of ATPS. As the time prolonged, polysaccharide in broken cells were released into the liquidmore easily. 35 min treatment was chosen because it was the most rational time, as the prolongation was not effective[11].

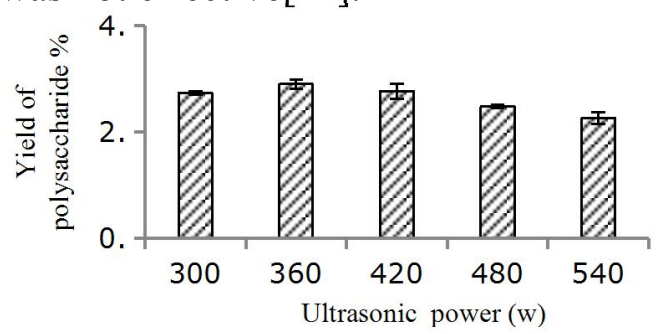

Figure.5. Effect of UP on yield of polysaccharide

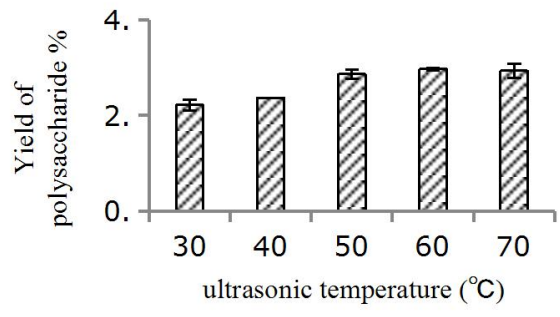

Figure.6. Effect of UTE on yield of polysaccharide

\section{Effect of UP}

As shown in Fig 5, when the power was increased from 300to $360 \mathrm{w}$, the yield of polysaccharide was increased and then decreased gradually when the power was over 360 w. It is known that the UP facilitates the destruction of cell walls. The results 
gained by Lucia Xavier indicated that most of polysaccharide in cell walls bereleased to the solvent at lower power, and higher power lead to the destruction of polysaccharides [10]. Therefore, the optimal UP was $360 \mathrm{w}$.

\section{Effect of UTE}

Temperature was considered at temperatures at $30,40,50,60$ and $70^{\circ} \mathrm{C}$. The results were esented in Fig 6. It indicated that the yield of polysaccharide was increased with increase in temperature from 30 to $60^{\circ} \mathrm{C}(\mathrm{p}<0.05)$, and then decreased when the temperature exceeded $60^{\circ} \mathrm{C}$. The results indicate that the high temperature could increase the solubility of polysaccharides. Besides, the yield is related to two main physical phenomena in UAE: acoustic cavitation and diffusion through the cell walls, and that the two phenomena were significantly enhanced by the temperature [11]. However, the structure of polysaccharidesis destroyed if the extraction temperature is too high. Also, high temperature may lead to the decrease of surface tension causing the damping of the ultrasonic wave. Thus, the temperature was selected as $60^{\circ} \mathrm{C}$.

\section{Optimization of Extraction Conditions and Analyses of RSM}

According to the single factor experiment, $11.5 \%$ PEG (6000) and 14\% $\left(\mathrm{NH}_{4}\right)_{2} \mathrm{SO}_{4}$ were chosen the study of the ultrasonic-assisted extraction. A BBD of RSM was conducted to optimize the conditions for extracting polysaccharide from hawthorn leaves using the UA-ATPS. Data were analyzed by analysis of variance, and factors were considered to be significantly different at $p<0.05$. The ANOVA results, the fit, and the adequacy of the models are summarized in Table 1 . The model $p$ - value of 0.0051 indicates that the model was significant, and lack of fit $(0.1392)$ indicates that the experimental results had a good fit with model. $p<0.05$ indicates that the factors have a significanteffect on the yield of polysaccharide. In this case, $\mathrm{X}_{2}, \mathrm{X}_{4}, \mathrm{X}_{1} \mathrm{X}_{2}$, $\mathrm{X}_{1} \mathrm{X}_{3}, \mathrm{X}_{1}^{2}, \mathrm{X}_{2}^{2}$ and $\mathrm{X}_{4}^{2}$ were all significant model terms.

$\mathrm{Y} 1=+2.871741+0.10775 * \mathrm{X}_{2}+0.119167 * \mathrm{X}_{4}-0.183069 * \mathrm{X}_{1} * \mathrm{X}_{1}+0.1955 * \mathrm{X}_{1} * \mathrm{X}_{2}-0.18425 * \mathrm{X}_{1} * \mathrm{X}_{3}-$ $0.087319 * \mathrm{X}_{2} * \mathrm{X}_{2}-0.085944 * \mathrm{X}_{4} * \mathrm{X}_{4}$

Table 1. ANOVA of RSM for the yield of polysaccharide

\begin{tabular}{cccccc}
\hline source & Sum of & df & Mean square & F value & P-valueProb $>$ F \\
\hline Model & 1.01 & 14 & 0.072 & 4.75 & 0.0051 \\
X1 & 0.036 & 1 & 0.036 & 2.40 & 0.1470 \\
X2 & 0.14 & 1 & 0.14 & 9.20 & 0.0104 \\
X3 & 0.034 & 1 & 0.034 & 2.23 & 0.1616 \\
X4 & 0.17 & 1 & 0.17 & 11.25 & 0.0057 \\
X1X2 & 0.15 & 1 & 0.15 & 10.09 & 0.0080 \\
X1X3 & 0.14 & 1 & 0.14 & 8.97 & 0.0112 \\
X1X4 & 0.016 & 1 & 0.016 & 1.05 & 0.3261 \\
X2X3 & 0.00001 & 1 & 0.00001 & 0.29 & 0.6016 \\
X2X4 & 0.012 & 1 & 0.012 & 0.82 & 0.3828 \\
X3X4 & 0.028 & 1 & 0.028 & 1.87 & 0.1960 \\
X1 ${ }^{2}$ & 0.25 & 1 & 0.25 & 16.76 & 0.0015 \\
X2 ${ }^{2}$ & 0.080 & 1 & 0.080 & 5.28 & 0.0404 \\
X3 ${ }^{2}$ & 0.059 & 1 & 0.059 & 3.90 & 0.0716 \\
X4 $^{2}$ & 0.078 & 1 & 0.078 & 5.16 & 0.0423 \\
Residual & 0.18 & 12 & 0.015 & & \\
Lack of fit & 0.18 & 10 & 0.018 & 6.57 & 0.1392 \\
\hline
\end{tabular}


The 3D response surface curves were shown in Fig 7. Each figure indicates the effects of two factors on the yield of polysaccharide while the other two factors were kept at zero level. Fig 7-a shows the interaction of ratio of material to solvent and UT, the UP and temperature was at zero level. The yield of polysaccharide was increased with the increase ratio of material to solvent and UT at the initial stage and then decreased when the ratio of material to solvent and UT beyond limits. Fig 7-b shows the interaction of ratio of material to solvent and UP when the UT and temperature were kept at zero level. The effects of ratio of material to solvent and UT were highly significant on yield of polysaccharide.
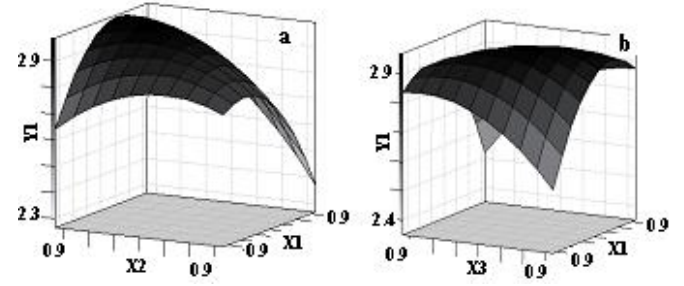

Fig.7. Response surface plots showing the effect of different extraction variables on the yield of polysaccharide (X1: ratio of material to solvent; X2: UT)

Byusing SAS 8.0 software, the optimum conditions for extracting polysaccharides from hawthorn leaves were ratio of material to solvent $1: 34.59$, UT $35.58 \mathrm{~min}$, UP 318.29 w, UTE $67.29^{\circ} \mathrm{C}$, respectively. The maximum predicted yield of polysaccharides was $3.05 \%$. To further testing the reliability of the experimental method, the extraction experiment was carried out by using the adjusted extraction conditions: ratio of material to solvent 1:34, UT35min, UP $300 \mathrm{w}$ and UTE $65^{\circ} \mathrm{C}$, and theyield of polysaccharide was $3.05 \%$.

\section{Conclusions}

In the present study, ATPS with $11.5 \%$ PEG and $14 \%\left(\mathrm{NH}_{4}\right)_{2} \mathrm{SO}_{4}$ was used as the extractionsolvent. Through the study of ultrasound extraction process, it was indicated that UT and UTE were the most important parameters. The optimum condition of extracting polysaccharide was ratio of material to solvent 1:34, UT $35 \mathrm{~min}$, UP $300 \mathrm{w}$ and UTE $65^{\circ} \mathrm{C}$, respectively. Under the most optimal conditions, the experimental yield of polysaccharide was $3.0 \%$. This result demonstrated the successful extraction of polysaccharide with ultrasonic-assisted ATPS extraction, providing potential benefits for industrial extraction of polysaccharide from hawthorn leaves.

\section{References}

[1] Han Junyan, Tan Dehong, Liu Guangchun. Hawthorn-A Health Food, Applied Mechanics and Material. 140 (2012) 350-354.

[2] Pengzhan Liu, HeikkiKallio, Baoru Yang. Phenolic Compounds in Hawthorn (Crataegusgrayana) Fruits and Leaves and Changes during Fruit Ripening, Food Chemistry. 59(2011) 11141-11149.

[3]YueQuan, Shuang Yang, Jie Wan, Tingting Su, Jing Zhang, Zhanyong Wang. Optimization for the extraction of polysaccharides from Nostoc commune and its antioxidant and antibacterial activities, Journal of the Taiwan Institute of Chemical Engineers. (2015) 1-8. 
[4] Ningbo Liao, JianjunZhong, Xingqian Ye, Shan Lu, Wenjun Wang, Ronghua Zhang, JianXu, ShiguoChen,Donghong Liu. Ultrasonic-assisted enzymatic extraction of polysaccharide from Corbiculafluminea: Characterization and antioxidant activity, LWT - Food Science and Technology. 60 (2015) 1113-1121.

[5] Chunjian Zhao, Zhao Li, Chunying Li, Lei Yang, Liping Yao, Yujie Fu, XinHe,Kunming Shi, Zhicheng Lu. Optimized extraction of polysaccharides from Taxuschinensisvar.mairei fruits and its antitumor activity, International Journal of Biological Macromolecules. 75 (2015) 192-198.

[6] Cai-ping Zhu, Xi-chuanZhai, Lin-qiang Li, Xiao-xia Wu, Bing Li. Response surface optimization of ultrasound-assisted polysaccharides extraction from pomegranate peel, Food Chemistry. 177 (2015) 139-146.

[7] Zhenyu Cheng, Haiyan Song, Yingjie Yang, Yan Liu, Zhigang Liu, Haobin Hu, Yang Zhang. Optimization of microwave-assisted enzymatic extraction of polysaccharides from the fruit of SchisandrachinensisBaill, International Journal of Biological Macromolecules. 76 (2015) 161-168.

[8]HongliangZeng, Yi Zhang, Shan Lin, YeyeJian, Song Miao, BaodongZheng.Ultrasonic-microwave synergistic extraction (UMSE) and molecular weight distribution of polysaccharides from Fortunella margarita (Lour.)Swingle, Separation and Purification Technology. 144 (2015) 97-106.

[9] Guo Y X, Han J, Zhang D Y, Wang L H, Zhou L L. An ammonium sulfate/ethanol aqueous two-phase system combined with ultrasonication for the separation and purification of lithospermic acid B from Salvia miltiorrhiza Bunge, UltrasonicsSonochemistry. 19 (2012) 719-724.

[10] Lucia Xavier, M. Sonia Freire, Isabel Vidal-Tato and Julia GonzalezAlvarez.Aqueous two-phase systems for the extraction of phenolic compounds from eucalyptus (Eucalyptus globulus) wood industrial wastes,Society of Chemical Industry. 89 (2014) 1772-1778.

[11] Zhi Ying, Xiaoxiang Han, Jianrong Li. Ultrasound-assisted extraction of polysaccharides from mulberry leaves, Food Chemistry. 127 (2011) 1273-1279. 